\title{
APPLICATIONS OF THE SEMISIMPLE SPLITTING
}

\author{
BY RICHARD TOLIMIERI
}

Communicated by L. Auslander, September 2, 1970

Let $S$ be a solvable simply connected analytic group. A closed subgroup $C$ of $S$ will be called uniform in $S$ if the quotient space $S / C$ is compact. We will make the assumption throughout the rest of the paper that a closed uniform subgroup $C$ of $S$ has the property that it contains no normal analytic subgroup of $S$. Let $C$ be a closed uniform subgroup of $S$. In [4], G. D. Mostow proved that the intersection of $C$ with the nil-radical of $S$ must be uniform in the nil-radical of $S$. L. Auslander in [6], [7] exploited this fact to relate the structure of $C$ to the structure of $S$. The object of this paper is to show how the semisimple splitting of $S$, introduced in [1] and studied further in [2] and [3], provides a convenient language with which to deal with the main theorems of [4], [6] and [7].

Preliminaries. We shall need the following ideas from the theory of algebraic groups. Let $X, Y$, and $Z$ be subgroups of $\operatorname{Gl}(n, R)$, the group of all $n$ by $n$ real matrices. We denote the algebraic hull of $X$ by $Q(X)$, and the group of commutators of $X$ and $Y$ by $(X, Y)$. Then

(a) $a((X, Y))=a((a(X), Q(Y)))$.

(b) If $X$ is a solvable algebraic group then we can write

$$
X=U \cdot T \quad \text { (semidirect product) }
$$

where $U$ is the collection of all unipotent matrices in $X$ and $T$ is a maximal completely reducible subgroup of $X$. Moreover if $T^{\#}$ is another maximal completely reducible subgroup of $X$ then there is an $x$ in $U$ such that $x T x^{-1}=T^{\#}$.

We will require also the following facts from the theory of nilpotent Lie groups. By a real nilpotent group $N$ we mean a simply connected nilpotent analytic group. We denote the Lie algebra of $N$ by $L(N)$. The exponential map defines a homeomorphism of $L(N)$ onto $N$. By a lattice of a vector space $V$ we mean the collection of all integer combinations of a basis of $V$. A discrete uniform subgroup $C$ of $N$ is called

AMS 1969 subject classifications. Primary 2050; Secondary 2048, 1450.

Key words and phrases. Algebraic group, the algebraic hull, unipotent matrix, semisimple matrix completely reducible group, nilpotent group, nil-radical solvable group, simply connected, lattice, discrete, uniform, semisimple splitting, Birkhoff embedding theorem, eigenvalues semidirect product, Fitting one-space, first cohomology group. 
a lattice of $N$ if it is the exponential of a lattice in $L(N)$. Given a discrete uniform subgroup $C$ of $N$ and a group $A$ of automorphisms of $N$ such that $A(C)=C$, there exists a lattice $C^{\#}$ of $N$ containing $C$ as a subgroup of finite index such that $A\left(C^{\sharp}\right)=C^{\sharp}$. We will also assume that the reader is familiar with the work of $\mathrm{Mal}^{\prime} \mathrm{cev}$ [5] whose results we will of ten state in the language of algebraic groups.

The semisimple splitting. Let $S$ be a simply connected solvable analytic group having nil-radical $H$. We will state those properties of the semisimple splitting of $S$ needed in what follows.

THEOREM 1. There exists a unique group $S^{\#}$ containing $S$ as a normal subgroup with the following properties:

(a) $S^{\#}$ has a semidirect product decomposition $N \cdot T$ where $N$ is the nil-radical of $S^{\sharp}$, and $T$ is an abelian analytic group acting faithfully on $N$ as a group of semisimple automorphisms. Any such decomposition of $S^{\#}, S^{\#}=N \cdot T$ will be called a $M a l^{\prime}$ cev decomposition of $S^{\#}$ and $T$ will be called a Mal'cev factor of $S^{\#}$. We denote the projection of $S^{\#}$ onto $N$ by $P_{T}$ and the projection of $S^{\#}$ onto $T$ by $t_{T}$.

(b) Let $S^{\#}=N \cdot T=N \cdot T^{\prime}$ be two $M a l^{\prime}$ cev decompositions of $S^{\#}$. Then there is an $x$ in $H$ such that $x T x^{-1}=T^{\prime}$.

(c) Let $S^{\#}=N \cdot T$ be a Mal'cev decomposition of $S^{\#}$. Then the restriction of $P_{T}$ to $S$ is a homeomorphism of $S$ onto $N$.

(d) $S$ and $N$ generate $S^{*}$.

(e) $H$ is contained in $N$ and $S^{\# /} H$ is abelian.

(f) There exists a faithful finite dimensional representation

$$
\beta: S^{\#} \rightarrow \mathrm{Gl}(n, R)
$$

such that $\beta(N)$ is the collection of all unipotent automorphisms in $\alpha\left(\beta\left(S^{*}\right)\right)$, and for any $M a l^{\prime}$ cev factor $T$ of $S^{\#}, \beta(T)$ is a group of semisimple matrices.

Statements (a)-(e) can be found in [1]. Statement (f) follows from the Birkhoff imbedding theorem and can be found in [9].

Let $C$ be a closed uniform subgroup of $S$. By (f) we lose no generality if we assume in all that follows that $S^{\#}$ is a matrix group. By (b) of the preliminary section we can write $a(C)=U_{C} \cdot T_{C}$ where $U_{C}$ is the collection of all unipotent matrices in $Q(C)$ and $T_{C}$ is a maximal completely reducible subgroup. Let $T^{\prime}$ be a maximal completely reducible subgroup of $Q(S)$ containing $T_{C}$. Since maximal completely reducible subgroups of $Q(S)$ are conjugate over $(S, S)$ we can find a $\mathrm{Mal}^{\prime} \mathrm{cev}$ factor $T$ of $S^{\#}$ such that $T \subset T^{\prime}$. Hence we can write a Mal'cev de- 
composition $S^{\sharp}=N \cdot T$ where $t_{T}(C) \subset Q(C)$. We shall say in this case that the Mal'cev decomposition $N \cdot T$ of $S^{\#}$ is compatible with $C$.

Mostow's theorem. We shall assume the following simple lemma. (See Lemma 6 in [4].)

LemMa 1. Let $\Delta$ be a lattice in a vector space $V$. Then there is an $\epsilon>0$ depending solely on the dimension of $V$ such that if $A$ is an automorphism of $V$ such that $A(\Delta)=\Delta$ and all the eigenvalues of $A$ are within $\epsilon$ of one, then $A=I$, where $I$ denotes the identity map on $V$.

LEMMa 2. Let $S$ be a simply connected solvable analytic group with nil-radical $H$. Then if $S$ has a discrete uniform subgroup $C$ such that $S=(H C)^{-}$, S is nilpotent. We are using a bar to the superior of a subgroup of $S$ to denote the closure of the subgroup in $S$.

Proof. Let $N \cdot T$ be a Mal'cev decomposition of $S^{\sharp}$ compatible with $C$. Let $M=Q(C \cap H)$ and $C^{\prime}=M C$. Since $C^{\prime} / C$ is compact, $C^{\prime}$ is a closed subgroup of $S$. Hence $P_{T}\left(C^{\prime}\right)$ is a closed uniform subgroup of $N$ that is invariant under $t_{T}\left(C^{\prime}\right)$. The connected component of the identity of $P_{T}\left(C^{\prime}\right)$ is $M$. Moreover $M$ is a normal subgroup of $S^{*}$. Since $T$ is completely reducible we must show that $\operatorname{ad}_{M} T=I$ and that $T$ induces the identity on $N / M$. If $\operatorname{ad}_{M} T \neq I$ then there is an element $c$ in $C$ such that $\operatorname{ad}_{M} c$ is not unipotent but has eigenvalues arbitrarily close to one. But $\operatorname{ad}_{M} C$ takes $C \cap H$ onto itself, hence we contradict Lemma 1 . Thus $\operatorname{ad}_{M} T=I$. Since $P_{T}\left(C^{\prime}\right) / M$ is a discrete uniform subgroup of $N / M,\left(C, P_{T}\left(C^{\prime}\right)\right) \subset M$ implies that $T$ induces the identity on $N / M$.

LEMMA 3. Let $S$ be a simply connected solvable analytic group with nil-radical $H$. Let $C$ be a closed uniform subgroup of $S$. Then the identity component $C_{0}$ of $C$ is contained in $H$. Moreover if $C / C_{0}$ is nilpotent, then $C$ is nilpotent.

Proof. Let $N \cdot T$ be a $\mathrm{Mal}^{\prime}$ cev decomposition of $S^{\#}$ compatible with $C$. Since $C$ is uniform in $S$ it follows that $N \subset Q(C)$. Hence $C_{0}$ is normalized by $N$ and $C_{0} \cap H$ is a normal analytic subgroup of $N$. Take $x$ in $C_{0}$ and write $x=n t$ where $n$ is in $N$ and $t$ is in $T$. Let $K$ be the ideal in $N$ generated by $(T-I) N$. Then $K$ is a normal analytic subgroup of $S^{\#}$ contained in $C_{0} \cap H$. Hence since we are assuming that $C_{0}$ contains no nontrivial normal analy tic subgroup of $S$ we have that $t=I$. Then $\operatorname{ad}_{H} C$ consists of unipotent automorphisms and $H C_{0}$ is a normal nilpotent analytic subgroup of $S$. Hence $C_{0} \subset H$. Assume now that $C / C_{0}$ is nilpotent. Then $Q(C) / C_{0}$ is nilpotent. Take $x$ in $C$ and write $x=n t$. 
Then $(t-I) N \subset C_{0} \cap H$ and we can argue as before to conclude that $C$ operates on $N$ by unipotent automorphisms. Hence $C$ is nilpotent.

THEOREM 2. Let $C$ be a closed uniform subgroup of a simply connected solvable analytic group $S$. Let $H$ be the nil-radical of $S$. Then $H C$ is a closed subgroup of $S$. Moreover $C_{0} \subset H$.

Proof. Let $G$ be the identity component of $(H C)^{-}$. We must show that $H=G$. Let $C^{\prime}=C \cap G$. Then $C^{\prime}$ is a closed uniform subgroup of $S$ and $S=\left(H C^{\prime}\right)^{-}$. Clearly $C_{0}$ is a normal subgroup of $G$. Hence by Lemma $2, G / C_{0}$ is nilpotent. Arguing as in Lemma 3, we can show that $\operatorname{ad}_{H} C^{\prime}$ consists of unipotent automorphisms. Hence $G$ is nilpotent.

Auslander's theorems. Let $Z^{n}$ denote the cartesian product of the integers taken $n$ times. Let $R^{n}$ denote the cartesian product of the reals taken $n$ times.

THEOREM 3. A necessary and sufficient condition for a simply connected solvable analytic group $S$ to contain $Z^{n}$ as a discrete uniform subgroup is that $S$ satisfy the diagram

$$
1 \rightarrow R^{s} \rightarrow S \rightarrow R^{t} \rightarrow 1
$$

where

(a) $s+t=n$,

(b) the extension is the split extension,

(c) the automorphisms of $R^{s}$ induced by $R^{t}$ form a compact group.

Proof. Assume that $S$ contains $Z^{n}$ as a discrete uniform subgroup. Let $N \cdot T$ be a Mal'cev decomposition of $S^{\#}$ compatible with $Z^{n}$. Since $P_{T}\left(Z^{n}\right)$ is a discrete uniform subgroup of $N$ centralized by $a\left(Z^{n}\right)$, we have that $Z^{n}$ is a discrete uniform subgroup of $N$. Hence $N$ is abelian and $T$ is compact. From $(N, T) \subset H$ and $T$ being completely reducible, it follows that there is a subspace $N^{\#}$ of $N$ such that $N=N^{\sharp} \oplus H$ as a vector space and $\left(T, N^{\sharp}\right)=(1)$. Let $S^{\prime}=P_{T}^{-1}\left(N^{\sharp}\right)$. Then $S=H \cdot S^{\prime}$ as a semidirect product.

The converse is trivial.

THEOREM 4. Let $S$ be a simply connected solvable analytic group and let $C$ be a closed uniform subgroup of $S$. Assume that $C / C_{0}$ is nilpotent. Then there is a real nilpotent group $N$ such that $C$ is contained in $N$ as a closed uniform subgroup, and a compact abelian analytic group $T$ of automorphisms of $N$ such that $S \subset N \cdot T$. In fact $S^{\#}=N \cdot T$.

Proof. Since $C / C_{0}$ is nilpotent, Lemma 3 implies that $C$ is nil- 
potent. Moreover if $N \cdot T$ is a $\mathrm{Mal}^{\prime} \mathrm{cev}$ decomposition of $S^{\#}$ compatible with $C$ we have also that $C \subset N$ as a closed uniform subgroup.

In terms of the semisimple splitting many cohomological arguments in solvable Lie theory depend upon the following lemma. (See Lemma 2.1 of [2].)

Lemma 4. Let $T$ be an abelian analytic group of semisimple automorphisms of a vector space $V$. Assume that the Fitting one-space of $T$ in $V$ is trivial. Then the first cohomology group vanishes, i.e. $H^{1}(T, V)$ $=0$.

The following theorem is easily seen to imply Theorem 2 of [7].

TheOREM 5. Let $N$ be a real nilpotent group and let $T_{i}, i=1,2$, be abelian analytic groups of semisimple automorphisms of $N$. Let $H$ be a normal analytic subgroup of $N$ invariant under $T, i=1,2$, such that,

(a) $N / H$ is abelian,

(b) $T_{i}, i=1,2$, induce the identity map on $N / H$,

(c) $T_{1}$ restricted to $H$ is equal to $T_{2}$ restricted to $H$. Let $\alpha: T_{1} \rightarrow T_{2}$ be the induced isomorphism.

(d) Let $M$ be the last term in the lower central series of $N$. Then the group of automorphisms on $N / M$ induced by $T_{1}$ and $T_{2}$ coincide.

Then there is an isomorphism $\bar{\alpha}$ of $N$ such that the map

$$
\bar{\alpha}: N \cdot T_{1} \rightarrow N \cdot T_{2}
$$

given by $\bar{\alpha}(n, t)=(\bar{\alpha}(n), \alpha(t))$ for $n$ in $N$ and $t$ in $T_{1}$, defines an isomorphism of the semidirect product $N \cdot T_{1}$ onto the semidirect product $N \cdot T_{2}$.

Proof. Choose $x$ in $N$ such that $(T, x)=(1)$. Consider the map $\eta: T_{2} \rightarrow M$ given by $t(x)=\eta(t) \cdot x$ for $t$ in $T_{2}$. Write $M=M_{1} \oplus M_{2}$ where $M_{1}$ is the Fitting one-space of $T$ and $M_{2}$ is a subspace of $M$ complementary to $M_{1}$ and invariant under $T_{2}$. Consider the maps $\eta_{i}: T_{2} \rightarrow M_{i}$, given by $\eta(t)=\eta_{1}(t) \eta_{2}(t)$. Then $\eta_{2}$ is a cocycle from $T_{2}$ to $M_{2}$. Thus there is an $h$ in $M_{2}$ such that $\eta_{2}(t)=(t, h)$ for all $t$ in $T_{2}$. Let $x^{\prime}=h^{-1} x$. Then it is easy to see that $\left(T_{2}, x^{\prime}\right)=(1)$. Now let $X$ be a subspace of $N$ such that $N=H \oplus X$, and $(T, X)=(1)$. Let $x_{i}, i=1, \cdots, n$, be a basis for $X$. Choose $h_{i}$ in $M$ such that $\left(T_{2}\right.$, $\left.h_{i}^{-1} x_{i}\right)=(1)$. Clearly there is an isomorphism $\bar{\alpha}$ of $N$ which maps $x_{i}$ onto $h_{i}^{-1} x_{i}$ and which acts by the identity map on $H$.

\section{REFERENCES}

1. L. Auslander and L. Green, G-induced flows, Amer. J. Math. 88 (1966), 43-60. MR 33 \#7456. 
2. L. Auslander and J. Brezin, Almost algebraic Lie algebras, J. Algebra 8 (1968), 295-313. MR 37 \#344.

3. R. Tolimieri, Foundations of solvable Lie groups, J. Algebra (to appear).

4. G. D. Mostow, Factor spaces of solvable groups, Ann. of Math. (2) 60 (1954), 1-27. MR 15, 853 .

5. A. Mal'cev, On a class of homogeneous spaces, Izv. Akad. Nauk SSSR Ser. Mat. 13 (1949), 9-32; English transl., Amer. Math. Soc. Transl. (1) 9 (1962), 276-307. MR 10, 507.

6. L. Auslander and M. Auslander, Solvable Lie groups and locally euclidean Riemann spaces, Proc. Amer. Math. Soc. 8 (1958), 933-941. MR 21 \#2021.

7. L. Auslander, Solvable Lie groups acting on nilmanifolds, Amer. J. Math. 82 (1960), 653-660. MR 23 \#A241.

8. - On a problem of Phillip Hall, Ann. of Math. (2) 86 (1967), 112-116. MR $36 \# 1540$.

9. G. Birkhoff, Representability of Lie algebras and Lie groups by matrices, Ann. of Math. 38 (1937).

Yale University, New Haven, Connecticut 06520 